\title{
A study of repeat dental general anaesthesia for children
}

\author{
Repeat general anaesthesia for paediatric dentistry by M. Harrison and L. Nutting BrDent J2000; 189:37-39
}

\section{Aims}

To investigate patterns of referral, disease and treatment for healthy children who had received two or more dental general anaesthetics (DGA) for exodontia.

\section{Methods}

Records from 200 episodes of repeat DGA were studied retrospectively.

\section{Results}

The mean age of patients at first referral was $5 \mathrm{y} 4 \mathrm{~m}$, and the mean interval before repeat was 22 months. Self-referrals rose from $14 \%$ at DGA1 to $30 \%$ at DGA2. New caries at second referral, where all diagnosed disease had been treated at DGA1, accounted for only $15 \%$ of the total. Where a specific request was made in the referral, only $8 \%$ of letters matched the charting made in hospital. $30 \%$ of all specific requests were honoured, but then required treatment for previously diagnosed caries at DGA2. 75\% of single-tooth extractions required repeat DGA for caries left at DGA1.

\begin{abstract}
Conclusions
It may be too optimistic to address only the most grossly diseased teeth when a child requires GA exodontia. A more radical treatment-planning approach, combining primary care, secondary care and public health considerations, may be required to avoid the unnecessary use of DGA.
\end{abstract}

\section{In brief}

Suggested strategies to reduce repeat DGA may include:

- Improved quality of referral letters

- More comprehensive treatment planning in pre-anaesthetic assessment clinics

- More explicit communication between the referrer and the provider of DGA

- Increased availability of specialist paediatric restorative care under the DGA

- Multi-agency targeting of parents for dental health education following DGA

\section{Comment}

Since the publication of the Poswillo $S$ report in 1990, the provision of dental general anaesthesia (DGA) has been in a state of flux. Following hard on the heels of a number of recent deaths associated with DGA, which have attracted considerable media attention, GDC guidance has significantly reduced the number of centres able to provide DGA. Few would disagree with the Poswillo's assertion that 'the use of general anaesthesia should be avoided wherever possible'. However, dental caries is still one of the most prevalent diseases in our child population, and DGA remains an appropriate treatment strategy for some children, especially the very young with multiple extensively carious teeth.

A key finding in this study was the high proportion of children who had been left with untreated dental caries at the first DGA returning for further DGA. The authors conclude that more aggressive pre- scribing of extractions at the pre-GA treatment planning stage may help to avoid this. The value of careful pre-GA planning by suitably trained dentists is emphasised, as is the importance of communication between the practitioners involved in the child's dental care.

However, these findings also suggest an apparent failure to adequately meet children's further treatment needs following DGA. In the vast majority of cases, second DGAs were needed for removal of teeth already identified as carious prior to the first DGA. It is reasonable to assume that the majority of such teeth were originally deemed to be potentially restorable. This highlights a common dilemma for those of us involved in providing DGA services for children: if carious teeth are not extracted who will restore them? Relatively few centres are able to offer comprehensive care (ie including restorations) under DGA and the recent reduction in the availability of
DGA does not yet appear to have been balanced by an increase in the availability of alternative strategies, such as advanced behaviour management and sedation. While many GDPs do make a genuine effort to provide appropriate care, successful treatment, especially for young patients, often exceeds the ability of nonspecialised dentists. A remuneration system which offers a fee for restoring a primary molar of around half that for the same restoration in a permanent tooth compounds the problem and effectively makes specialised paediatric dental practice within the NHS non-viable.

This timely paper prompts us to re-evaluate how effectively the dental needs of young children with extensive caries are currently being met.

\section{Stephen A. Fayle}

Consultant in Paediatric Dentistry,

Leeds Dental Institute 